\title{
Network meta-analysis of treatment regimens for inoperable advanced hepatocellular carcinoma with portal vein invasion
}

This article was published in the following Dove Press journal:

Therapeutics and Clinical Risk Management

Ming-Feng $\mathrm{Li}^{1,2, *}$

Henry WC Leung ${ }^{3, *}$

Agnes LF Chan ${ }^{4}$

Shyh-Yau Wang ${ }^{2, *}$

'Department of Medical Imaging, Buddhist Tzu-Chi General Hospital, Hualien, Taiwan; ${ }^{2}$ Department of Radiology, An-Nan Hospital, China Medical University, Tainan, Taiwan; ${ }^{3}$ Department of Radiation Oncology, An-Nan Hospital, China Medical University, Tainan, Taiwan; ${ }^{4}$ Department of Pharmacy, An-Nan Hospital, China Medical University, Tainan, Taiwan

*These authors contributed equally to this work
Correspondence: Shyh-Yau Wang Department of Radiology, An-Nan Hospital, China Medical University, No 66, Section 2, Changhe Road, Annan District, Tainan, Taiwan Tel +8866355 31II

Email d23930@mail.tmanh.org.tw

Henry WC Leung Department of Radiation Oncology, An-Nan Hospital, China Medical University, No 66, Section 2, Changhe Road, Annan District, Tainan, Taiwan Tel +886 63553 II I Email hwclmd@yahoo.com
Purpose: We assessed the efficacy and safety of different modalities using the network meta-analysis for inoperable hepatocellular carcinoma (HCC) with portal vein invasion. The interested modalities included stereotactic body radiotherapy (SBRT) combined with transarterial chemoembolization (TACE), three-dimensional radiotherapy (3D-RT) combined with hepatic arterial infusion chemotherapy (HAIC) or TACE, TACE plus sorafenib, and use of SBRT, HAIC, sorafenib, and TACE alone.

Methods: PubMed and Cochrane Library electronic databases were systematically searched for eligible studies published up to June 2017. We used network meta-analysis to compare the disease control rate (DCR) and severe adverse events for the eight interested regimens included in this analysis. Study quality was assessed following the Grading of Recommendations, Assessment, Development and Evaluations method.

Results: Fifteen studies published between 2010 and 2016 involving a total of 2,359 patients were enrolled in this network meta-analysis. With indirect comparison of DCR and overall safety, the pooled results showed that RT plus HAIC was the most effective regimen in treating advanced $\mathrm{HCC}$ with portal vein tumor thrombosis, followed by RT plus TACE. HAIC alone and sorafenib combined with HAIC appeared least effective intervention regimens. The incidence of treatment-related adverse events of grade 3 or 4 occurred less in the patients who received SBRT alone compared with other interested regimens.

Conclusion: 3D-RT combined with HAIC or TACE showed more favorable treatment responses compared with other regimens in advanced HCC patients with portal vein tumor thrombosis.

Keywords: SBRT, HAIC, 3D-RT plus HAIC, sorafenib, network meta-analysis, PVTT

\section{Introduction}

Hepatocellular carcinoma (HCC) is the second leading cause of cancer-related death in Taiwan and worldwide in 2016 and 2015. ${ }^{1}$ Most HCCs are diagnosed at an advanced stage, and surgical complete resection is not suitable, in particular for patients with portal vein tumor thrombus (PVTT). ${ }^{2-4}$ Those with PVTT are at an increased risk of liver failure by wide dissemination of tumor throughout the liver. ${ }^{5,6}$ The prognosis is extremely poor, and the patients have lower tolerance to treatment with limited survival about only several months. ${ }^{7,8}$

In recent years, various treatment modalities such as transarterial chemoembolization (TACE), hepatic arterial infusion chemotherapy (HAIC), three-dimensional radiotherapy (3D-RT), stereotactic body radiotherapy (SBRT), immunotherapy, and sorafenib have been tried for the treatment of advanced HCC with PVTT, but the optimal treatment strategy remains controversial. Recently, some studies 
reported that TACE or repetitive HAIC in advanced HCC has achieved favorable results. ${ }^{9-13}$ With the advances in radiation technology, 3D-RT or SBRT has been used for advanced HCC with the benefit of using higher radiation doses directed at the tumor that spare the organ at risk from substantial radiation. ${ }^{14,15}$ Many studies have reported that 3D-RT plus TACE or HAIC showed good tumor and PVTT response than TACE or HAIC used alone. ${ }^{16-21}$ However, some studies indicated that adding RT to TACE or HAIC does not improve survival but increases the incidence of adverse events compared with TACE or HAIC alone. ${ }^{19,22}$ Therefore, the purpose of this network meta-analysis was to evaluate the efficacy and safety of these regimens in terms of disease control rate (DCR) and severe adverse events in advanced HCC patients with PVTT.

\section{Methods}

\section{Literature search}

We conducted this network meta-analysis in accordance with the Preferred Reporting Items for Systematic Reviews and Meta-Analyses statement. ${ }^{23}$ A systematic literature search of the PubMed and Cochrane Library from inception through November 2017 was performed. The search strategy was based on combinations of the following keywords: ("advanced" [Mesh]) AND ("hepatomas" or "liver cell carcinomas" [MeSH terms]) OR ("hepatocellular carcinoma" [MeSH terms] AND [all fields]) AND ("clinical study" [publication type] OR “cohort as topic" [MeSH terms]) AND ("sorafenib" or "Nexavar") AND ("hepatic arterial infusion chemotherapy" or "HAIC") AND ("TACE" or "transarterial chemoembolization") AND ("radiotherapy" or "stereotactic body or SBRT"). In addition, we manually examined the titles of all references within the selected articles to identify other potentially appropriate articles. Two authors (AC and HL) evaluated the titles and abstracts independently. Disagreements were discussed until consensus was reached. Letters to the editor, case reports, nonrandomized trials, animal studies, editorials, and posters were excluded. The language was also restricted to English.

\section{Study selection criteria}

The selected studies had to meet the following criteria: 1) nonrandomized or cohort studies; 2) included patients with pathologically proven advanced inoperable HCC with PVTT; 3) detailed data on method, characteristics of patient population, tumor response rates, adverse events, and overall survival; 4) compared at least two arms that consisted of the abovementioned interested regimens; and 5) tumor response rates were defined and evaluated based on the comparison of abdominal computed tomography or magnetic resonance imaging before and after treatment according to the modified Response Evaluation Criteria in Solid Tumors (RECIST) guidelines for HCC. ${ }^{24}$ A complete response (CR) was defined as disappearance of all target/nontarget lesions after treatment; partial response (PR) was defined as follows: the size of lesion decreased $30 \%$ in the sum of the longest diameters of the target lesions; progressive disease (PD) was defined as follows: the size of lesion increased $20 \%$ in the sum of the longest diameters of the target lesions; stable disease (SD) was defined as not meeting the criteria of CR, PR, or PD, all other variations.

\section{Data extraction and quality assessment}

Two authors (AC and HL) independently reviewed and screened all eligible studies based on the study selection criteria detailed above. The following data were extracted and summarized in a standardized table, including the study's first author; characteristics of the population; and data to define advanced HCC, interventions, and outcomes such as overall survival and tumor response in terms of DCR and adverse events (Table 1). The assessment primary outcome in this study was DCR. The secondary outcomes were median overall survival and grade 3 or 4 adverse events. The DCR was defined as the percentage of a CR, PR, or SD for each study according to the modified RECIST guidelines.

The quality of the included studies was assessed using the Cochrane risk of bias tool (Version 5.1.0). Each study was evaluated independently by two authors explicitly with the following judgment system: low risk of bias, high risk of bias, or unclear (either lack of information or uncertainty for bias). ${ }^{25}$ The quality of evidence in these studies was evaluated by using the Grading of Recommendations, Assessment, Development and Evaluations (GRADE) profiler (GRADEpro) software, Version 3.2 (the Cochrane Information Management System). ${ }^{26}$

\section{Network meta-analysis}

We conducted a network meta-analysis to compare the outcomes among the 15 studies for advanced HCC with PVTT, which included direct (ie, head-to-head) and indirect treatment comparisons. ${ }^{19-22,27-37}$ We extracted the DCR and grade 3 or 4 adverse events data directly from the studies to calculate the odds ratios (ORs) with $95 \%$ confidence intervals (95\% CIs). A network meta-analysis was conducted using WinBUGS within Microsoft Excel (Microsoft Corporation, Redmond, WA, USA) to synthesize direct and indirect 


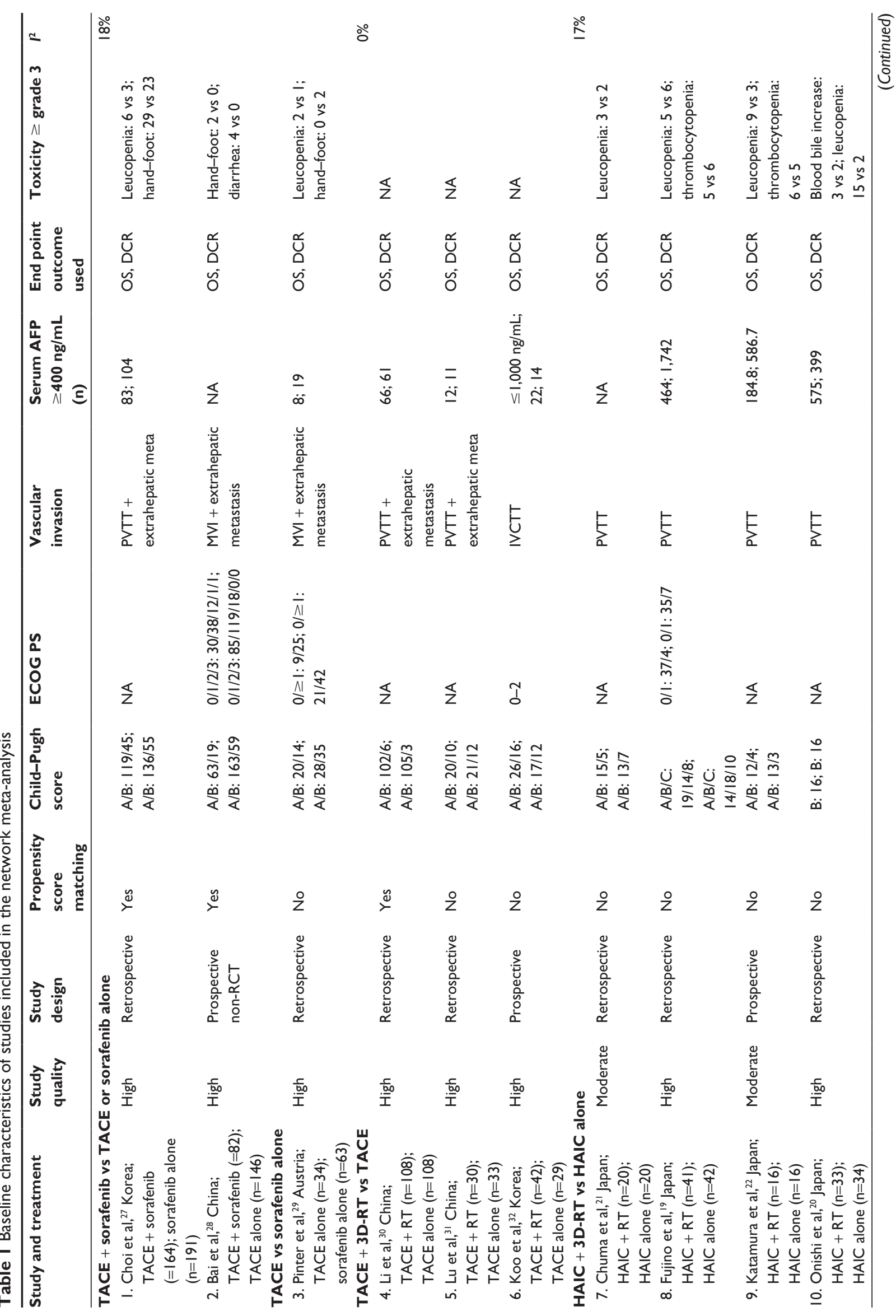




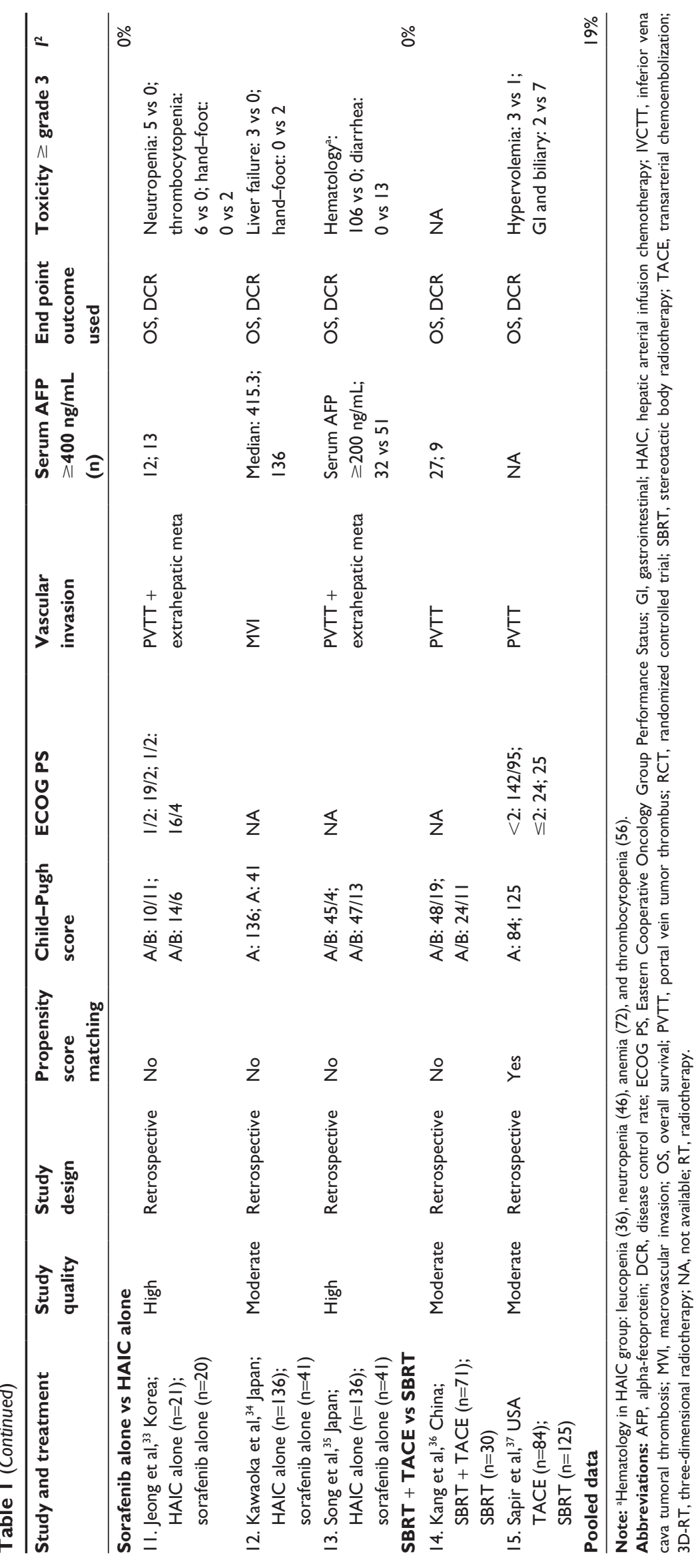


treatment regimens simultaneously and summarize all possible pairwise comparisons between the various regimens as a league table. ${ }^{38,39}$ The probability of each treatment for a particular outcome would be calculated and ranked first, second, third, etc. by the surface under the cumulative ranking (SUCRA) curve. ${ }^{40}$ The larger SUCRA number represented the best treatment regimens among the network meta-analysis.

\section{Statistical analysis}

The heterogeneity was assessed with the $I^{2}$ statistic using Review Manager (RevMan) software, Version 5.3.5 (The Cochrane Collaboration, Copenhagen, Denmark). ${ }^{45} I^{2}$ statistics were used to measure statistical heterogeneity. $I^{2}$ is the percentage of total variation attributable to differences observed between the studies rather than sampling error. It is also an intuitive and a simple expression of the inconsistency of studies' results. $I^{2}$ values $<25 \%$ and $>75 \%$ were regarded as indications of low and high heterogeneity, respectively. ${ }^{41}$ If significant heterogeneity existed, a fixed-effect statistical model would be used.

The assessment of network inconsistency was conducted by comparing the deviance residuals and deviance information criterion statistics in fitted consistency and inconsistency models to identify inconsistency presented in any loops in the treatment network. ${ }^{42}$ Inconsistency is caused by imbalances in effect modifiers from study to study, particularly in the direct and indirect evidence. We could select the points on the inconsistency plot to identify which study and treatment contributed to the network inconsistency.

The sensitivity analysis was performed to test the robustness of the network analysis by repeating the main computations using a random-effects model.

\section{Results}

\section{Characteristics of the selected studies}

A total of 222 relevant studies were identified through the comprehensive search. After all titles and abstracts were reviewed, 116 studies were excluded because of not matching with the selection criteria. After screening of full-length articles, we found 15 studies that matched the inclusion criteria. ${ }^{19-22,27-37}$ Twelve studies were retrospective cohort study, and three studies were prospective studies. ${ }^{22,28,32}$ All the studies were conducted in Asia, except two studies were in Austria and the USA. Table 1 presents the study characteristics. Figure 1 is the flow diagram of the systematic literature search and selection. A total of 2,359 patients were included in the network meta-analysis, among which 1,061 patients and 319 patients were treated with TACE and HAIC alone.

\section{Quality assessment}

Figure 2 summarizes the risk of bias for the 15 selected studies. All the studies selected exposed cohorts from the same population. Ten studies $(66.7 \%)$ showed a high risk of bias for the item of comprehensive matching or adjustment for those prognostic variables. Of these 10 studies, five studies (33.3\%) showed a high risk of bias for assessment criteria 2 (assessment of exposure) and five studies (33.3\%) showed a high risk of bias for the assessment of the presence or absence of prognostic factors. Therefore, the quality of evidence was assessed for the individual studies as moderate $22,33,34,36,37$ or high $^{12-20,27-32}$ according to the GRADE method (Table 1).

\section{Network meta-analysis}

The ranking table in Figure 3 shows the comparison of the efficacy of all treatment regimens against each other in terms of DCR in treating advanced HCC with portal vein invasion. Regimen in the left side was the most effective $(\mathrm{OR}>1)$, and regimen in the right side was the least effective $(\mathrm{OR}<1)$. From the ranking table generated, it was clear that RT plus HAIC would be the most effective in treating patients with advanced $\mathrm{HCC}$ and portal vein invasion (OR range: 4.52-6.40; 95\% CI =31.29-9.16 and 2.98-14.03), followed by RT plus TACE (OR range: $3.55-5.03,95 \% \mathrm{CI}=1.38-9.16$ and 2.18-11.82). HAIC alone and sorafenib combined with HAIC appeared the least effective, although it still showed significantly increased DCR when compared with other four regimens. We also compared the efficacy of all interested regimens in terms of the median overall survival retrieved from the included studies. RT plus TACE and RT plus HAIC were still the most effective in treating advanced $\mathrm{HCC}$ with portal vein invasion (Figure 4).

The results of SUCRA curve for each treatment in terms of DCR demonstrated the same results that 3D-RT combined with HAIC was the best regimen (SUCRA 0.9337) followed by 3D-RT combined with TACE (SUCRA 0.85). Sorafenib alone was the worst (SUCRA 0.1122) regimen for $\mathrm{mHCC}$ patients with portal vein invasion (Figure 5).

In the safety analysis, retrieving grade 3 or 4 adverse events from all included studies was limited. From the ranking table generated, it was found that regimens with OR $<1$ would be the safer regimens when compared with every other modalities (Figure 6). Then, SBRT, sorafenib, or TACE used alone had significant fewer severe adverse events as compared to other regimens. In the subgroup analysis, 


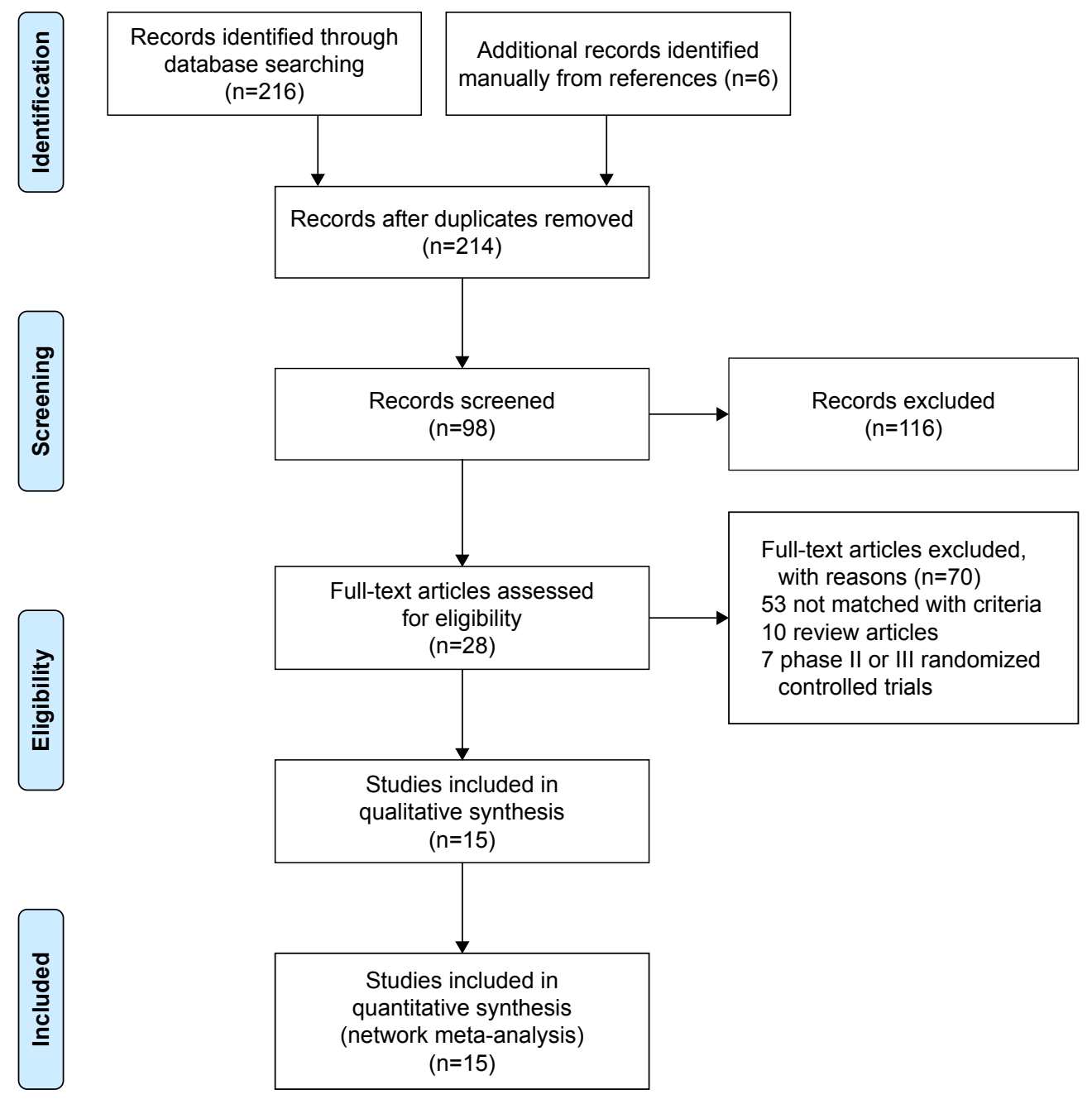

Figure I Flow chart diagram of searching strategy.

the severe hematological adverse events (grade 3 or 4 ) occurred in patients treated with 3D-RT combined with TACE or HAIC and TACE or HAIC used alone were leucopenia and thrombocytopenia. Hand-foot syndrome was the most common severe adverse event in sorafenib regimen (Table 1). However, three studies did not have grade 3 or 4 adverse events data available for analysis..$^{30-32}$

\section{Assessment of inconsistency and heterogeneity}

The assessment of inconsistency in the network meta-analysis was presented by the inconsistency plot in fixed-effects model. From the inconsistency plot in Figure 7, it was found that all studies were along with the line of equality, which may indicate that the effect modifiers are more evenly balanced across the network; it is more likely to be no potential inconsistency between different treatment modalities.

From the results of pooled data for heterogeneity $\left(I^{2}\right)$, it was found that between-studies variation within compared treatment modalities was 19\%. In the subgroup analysis of included treatment regimens, the heterogeneity $\left(I^{2}\right)$ values for TACE plus sorafenib versus TACE or sorafenib alone and HAIC + RT versus HAIC alone were $20 \%$ and $19 \%$, respectively, which indicated low heterogeneity $\left(I^{2}<25 \%\right)$. The heterogeneity $\left(I^{2}\right)$ for other regimens was $0 \%$ (Table 1 ).

The sensitivity analysis showed that the network results of the random-effects model were consistent with that of the fixed-effects model. The pooled OR remained unchanged in heterogeneity $\left(I^{2}=19\right)$ and slightly changed in OR $(1.96$ $[1.51,2.56]$ in random-effects model; $1.99[1.58,2.50]$ in fixed-effects model).

\section{Discussion}

To the best of our knowledge, this is the first network metaanalysis to compare the efficacy and safety of the treatment regimens in patients with advanced HCC and portal vein invasion. PVTT is a well-known poor prognostic factor for HCC patients, whose median survival time was only 2.7 months. ${ }^{43}$ 


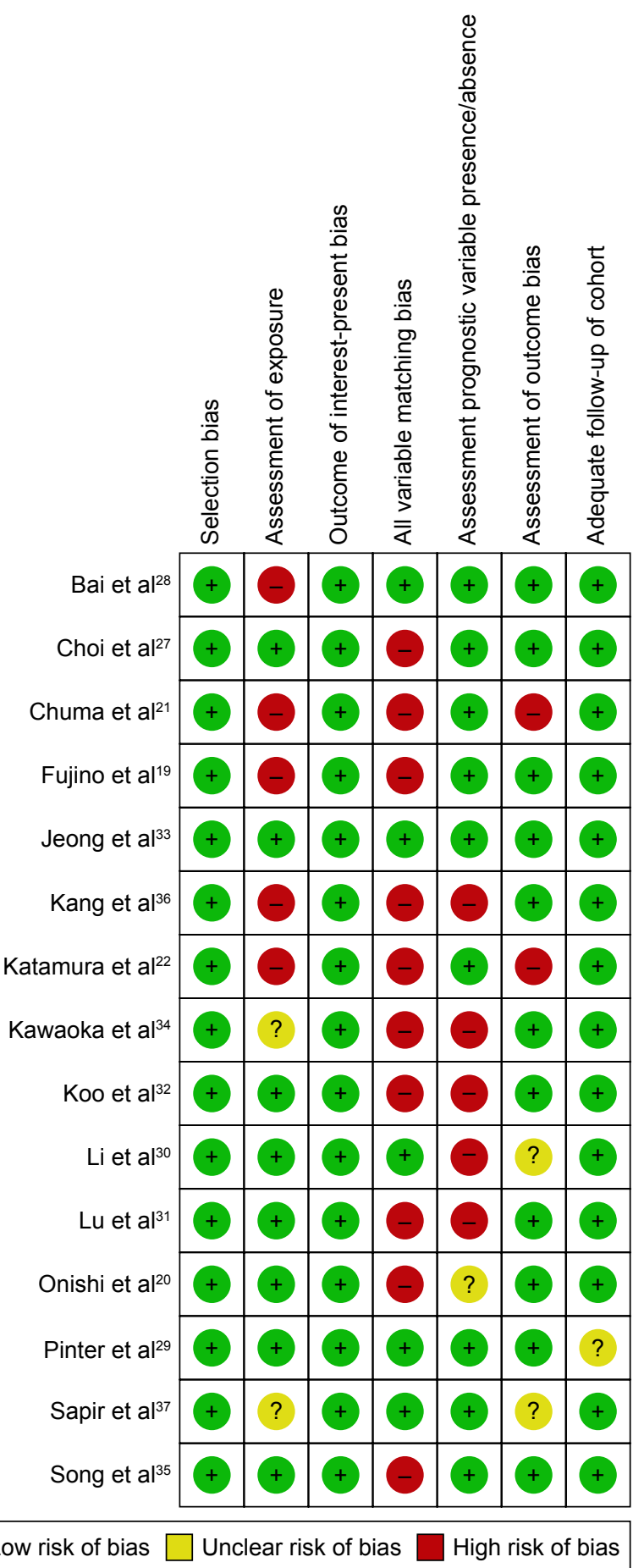

Figure 2 Risk of bias.

In this network meta-analysis, we collected the direct and indirect comparative data and assessed the tumor control rate, median survival rate, and severe adverse events in advanced HCC patients with PVTT undergoing different treatment modalities. The pooled results demonstrated that, in patients with advanced HCC with PVTT, those who were treated with local control combination regimens, such as 3D-RT plus TACE $\left(\mathrm{OR}_{\mathrm{RT}+\mathrm{TACE}}=3.55-5.03 ; 95 \% \mathrm{CI}=1.38-9.16\right.$ and $\left.2.98-11.82\right)$ and 3D-RT plus HAIC $\left(\mathrm{OR}_{\mathrm{RT}+\text { HAIC }}=4.52-6.40 ; 95 \% \mathrm{CI}\right.$ $=31.29-9.16$ and 2.98-14.03), could reach significantly higher PVTT and tumor response as well as better median overall survival $\left(\mathrm{OR}_{\mathrm{RT}+\mathrm{TACE}}=2.90-3.47 ; 95 \% \mathrm{CI}=2.02-4.17\right.$ and $\left.1.45-8.25 ; \mathrm{OR}_{\mathrm{RT}+\mathrm{HAIC}}=3.44 ; 95 \% \mathrm{CI}=1.58-7.55\right)$ compared with those other six regimens. The combination regimens were well tolerated with statistically nonsignificant and significant severe grade 3 or 4 adverse events $\left(\mathrm{OR}_{\mathrm{TACE}}+\right.$ Sorafenib $=0.32$; $95 \% \mathrm{CI}=0.07-1.34 ; \mathrm{OR}_{\mathrm{RT}+\text { HAIC }}=0.91 ; 95 \% \mathrm{CI}=0.44-1.89$; $\left.\mathrm{OR}_{\mathrm{RT}+\mathrm{TACE}}=0.07 ; 95 \% \mathrm{CI}=0.01-0.3\right)$. Based on these results, it was suggested that either TACE or HAIC in combination with 3D-RT is a better choice for advanced HCC patients with PVTT. No network meta-analysis has previously been published focusing on comparing different treatment modalities for patients with advanced HCC and portal vein invasion. Therefore, we compared the results of HAIC or TACE or sorafenib combined with 3D-RT with those from conventional meta-analyses published recently. We found that the results in our analysis may be considered as a further evidence to support the previous three conventional meta-analyses because the conventional meta-analysis conducted by Zhang et a ${ }^{45}$ and Huo and Eslick ${ }^{44}$ did not include all patients with portal vein invasion and the outcomes were only presented in terms of objective response rate. Thus, their results may be overestimation. ${ }^{44,45}$ Only the meta-analysis conducted by Zhao et al was similar to our study to enroll patients with advanced HCC with portal vein invasion. ${ }^{46}$

Various treatment modalities have been tried to treat advanced HCC patients with PVTT, but the optimal treatment remains controversial. Surgical resection has been considered as the first preferred modality for highly selected patients with reserved liver function and localized PVTT in the distal branch. TACE is considered as a first-line treatment for patients with HCC, Barcelona Cancer Clinic Liver Cancer (BCLC) stage B with an excellent liver function, and asymptomatic multinodular tumors without macroscopic vascular invasion or extrahepatic spread. HAIC has been used for advanced $\mathrm{HCC}$ without distant metastasis with improved survival in Asian countries, such as Japan, Korea, and China. SBRT is a newer treatment modality for patients with localized HCC and not eligible to TACE. ${ }^{47}$ 3D-RT and SBRT are emerging as practical treatment modalities for localized HCC. Although the BCLC guideline recommended sorafenib as the standard systematic therapy for advanced HCC patients, and sorafenib alone has limited efficacy for HCC patients with limiting extrahepatic spread. With the advances in the technology of radiation therapy, SBRT is a treatment option with local control rates of $75 \%$ to $100 \%$ at $1-2$ years of survival. ${ }^{48,49}$ Recently, many studies demonstrated that 


\section{OR $>1$ means the treatment in top left is better}

\begin{tabular}{|c|c|c|c|c|c|c|c|}
\hline RT HAIC & & & & & & & \\
\hline $\begin{array}{c}1.28 \\
(0.40-4.00)\end{array}$ & TACE + RT & & & & & & \\
\hline $\begin{array}{c}1.71 \\
(0.97-3.07)\end{array}$ & $\begin{array}{c}1.34 \\
(0.50-3.64)\end{array}$ & HAIC & & & & & \\
\hline $\begin{array}{c}2.65 \\
(1.04-6.88)\end{array}$ & $\begin{array}{c}2.08 \\
(0.99-4.50)\end{array}$ & $\begin{array}{c}1.55 \\
(0.74-3.31)\end{array}$ & Sorafenib TACE & & & & \\
\hline $\begin{array}{c}3.83 \\
(0.69-22.62)\end{array}$ & $\begin{array}{c}3.00 \\
(0.66-14.29)\end{array}$ & $\begin{array}{c}2.24 \\
(0.44-11.87)\end{array}$ & $\begin{array}{c}1.44 \\
(0.33-6.78)\end{array}$ & TACE + SBRT & & & \\
\hline $\begin{array}{c}4.52 \\
(1.29-15.87)\end{array}$ & $\begin{array}{c}3.55 \\
(1.38-9.16)\end{array}$ & $\begin{array}{c}2.63 \\
(0.87-7.99)\end{array}$ & $\begin{array}{c}1.70 \\
(0.68-4.18)\end{array}$ & $\begin{array}{c}1.19 \\
(0.33-3.79)\end{array}$ & SBRT & & \\
\hline $\begin{array}{c}5.36 \\
(1.99-14.55)\end{array}$ & $\begin{array}{c}4.17 \\
(2.43-7.62)\end{array}$ & $\begin{array}{c}3.12 \\
(1.40-7.10)\end{array}$ & $\begin{array}{c}2.01 \\
(1.23-3.31)\end{array}$ & $\begin{array}{c}1.40 \\
(0.32-5.64)\end{array}$ & $\begin{array}{c}1.18 \\
(0.56-2.57)\end{array}$ & TACE & \\
\hline $\begin{array}{c}6.40 \\
(2.98-14.03)\end{array}$ & $\begin{array}{c}5.03 \\
(2.18-11.82)\end{array}$ & $\begin{array}{c}3.73 \\
(2.25-6.35)\end{array}$ & $\begin{array}{c}2.41 \\
(1.41-4.13)\end{array}$ & $\begin{array}{c}1.67 \\
(0.34-7.76)\end{array}$ & $\begin{array}{c}1.42 \\
(0.54-3.81)\end{array}$ & $\begin{array}{c}1.20 \\
(0.64-2.22)\end{array}$ & Sorafenib \\
\hline
\end{tabular}

Figure 3 Comparisons of efficacy in terms of disease control rate in advanced HCC scheme.

Note: The numbers in bold indicate OR and $95 \% \mathrm{Cl}$ are significant.

Abbreviations: HAIC, hepatic arterial infusion chemotherapy; OR, odds ratio; SBRT, stereotactic body radiotherapy; TACE, transarterial chemoembolization; $\mathrm{RT}$, radiotherapy.

the combination of 3D-RT or SBRT with HAIC or TACE and TACE combined with sorafenib to treat PVTT were superior to any single treatment option. ${ }^{44}$ The mechanisms of the combination of radiotherapy (SBRT or 3D-RT) and
TACE may involve 1) the reduction of the radiation target volume and the increase of radiation dose precisely delivered to the target lesion and thus sparing uninvolved normal liver tissue; 2) the administration of RT after TACE may

\section{OR $>1$ means the treatment in top left is better}

\begin{tabular}{|c|c|c|c|c|c|c|c|}
\hline TACE + RT & & & & & & & \\
\hline $\begin{array}{c}1.01 \\
(0.31-3.26)\end{array}$ & RT HAIC & & & & & & \\
\hline $\begin{array}{c}2.34 \\
(0.75-7.33)\end{array}$ & $\begin{array}{c}2.32 \\
(0.48-11.06)\end{array}$ & TACE + SBRT & & & & & \\
\hline $\begin{array}{c}2.53 \\
(0.92-7.09)\end{array}$ & $\begin{array}{c}2.51 \\
(1.43-4.47)\end{array}$ & $\begin{array}{c}1.09 \\
(0.26-4.70)\end{array}$ & HAIC & & & & \\
\hline $\begin{array}{c}2.90 \\
(2.02-4.17)\end{array}$ & $\begin{array}{c}2.88 \\
(0.94-8.80)\end{array}$ & $\begin{array}{c}1.24 \\
(0.42-3.65)\end{array}$ & $\begin{array}{c}1.14 \\
(0.44-2.97)\end{array}$ & TACE & & & \\
\hline $\begin{array}{c}3.05 \\
(1.40-6.64)\end{array}$ & $\begin{array}{c}3.02 \\
(0.87-10.56)\end{array}$ & $\begin{array}{c}1.30 \\
(0.36-4.71)\end{array}$ & $\begin{array}{c}1.20 \\
(0.40-3.65)\end{array}$ & $\begin{array}{c}1.05 \\
(0.53-2.11)\end{array}$ & Sorafenib TACE & & \\
\hline $\begin{array}{c}3.02 \\
(1.45-6.36)\end{array}$ & $\begin{array}{c}3.01 \\
(0.82-10.85)\end{array}$ & $\begin{array}{c}1.29 \\
(0.55-3.08)\end{array}$ & $\begin{array}{c}1.19 \\
(0.37-3.79)\end{array}$ & $\begin{array}{c}1.04 \\
(0.55-2.00)\end{array}$ & $\begin{array}{c}0.99 \\
(0.38-2.53)\end{array}$ & SBRT & \\
\hline $\begin{array}{c}3.47 \\
(1.45-8.25)\end{array}$ & $\begin{array}{c}3.44 \\
(1.58-7.55)\end{array}$ & $\begin{array}{c}1.48 \\
(0.39-5.71)\end{array}$ & $\begin{array}{c}1.36 \\
(0.80-2.35)\end{array}$ & $\begin{array}{c}1.20 \\
(0.54-2.63)\end{array}$ & $\begin{array}{c}1.14 \\
(0.43-2.98)\end{array}$ & $\begin{array}{c}1.15 \\
(0.41-3.18)\end{array}$ & Sorafenib \\
\hline
\end{tabular}

Figure 4 Comparisons of efficacy in terms of median OS in advanced HCC.

Note: The numbers in bold indicate OR and $95 \% \mathrm{Cl}$ are significant.

Abbreviations: HAIC, hepatic arterial infusion chemotherapy; OR, odds ratio; OS, overall survival; SBRT, stereotactic body radiotherapy; TACE, transarterial chemoembolization; RT, radiotherapy. 


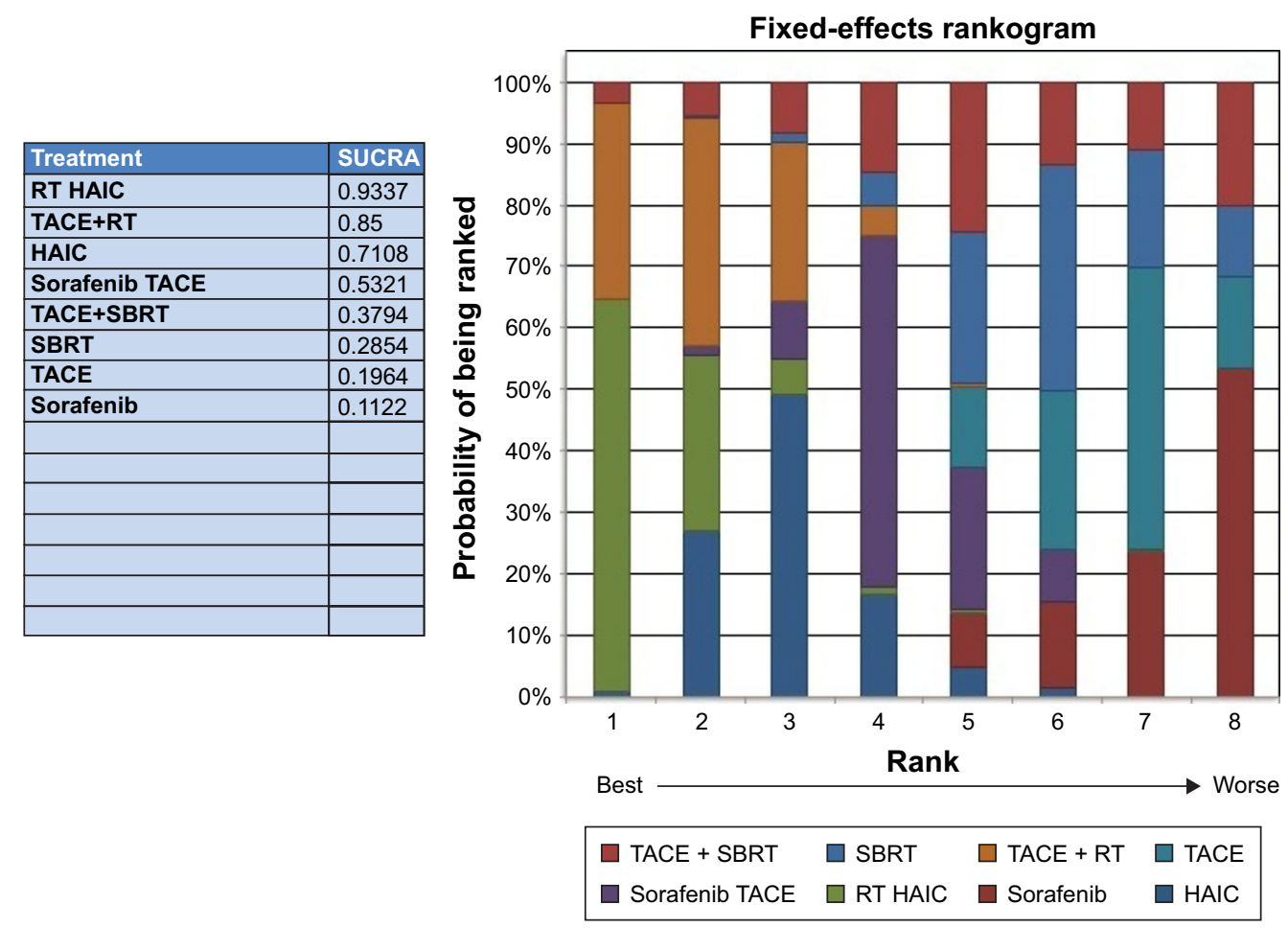

Figure 5 Rankogram of interested treatment modality.

Abbreviations: HAIC, hepatic arterial infusion chemotherapy; SBRT, stereotactic body radiotherapy; TACE, transarterial chemoembolization; RT, radiotherapy.

contribute to killing or inhibiting any residual tumor cells after TACE; $;^{31,36}$ and 3) PVTT is sensitive to RT, which can boost the treatment responses of tumors and PVTT, ${ }^{50,51}$ As for the mechanisms of combination of TACE and sorafenib, this multimodality may involve the embolization of the hepatic artery and reduce the portal vein blood supply to HCC..$^{52,53}$ Sorafenib is an orally active Raf kinase inhibitor with effects on tumor cell proliferation and tumor angiogenesis that acts by inhibiting the serine-threonine kinases Raf-1 and B-Raf. It also inhibits vascular endothelial growth factor receptors 1,2 , and 3 ; platelet-derived growth factor receptor $\beta$; and receptor tyrosine kinase receptor tyrosine kinases. ${ }^{54}$

The network meta-analysis is a useful method for incorporating information from both direct and indirect treatment

\begin{tabular}{|c|c|c|c|c|c|}
\hline SBRT & & & & & \\
\hline $\begin{array}{c}0.65 \\
(0.18-2.29)\end{array}$ & Sorafenib & & & & \\
\hline $\begin{array}{c}0.58 \\
(0.23-1.44)\end{array}$ & $\begin{array}{c}0.89 \\
(0.37-2.16)\end{array}$ & TACE & & & \\
\hline $\begin{array}{c}0.31 \\
(0.08-1.13)\end{array}$ & $\begin{array}{c}0.48 \\
(0.28-0.79)\end{array}$ & $\begin{array}{c}0.53 \\
(0.21-1.35)\end{array}$ & Sorafenib TACE & & \\
\hline $\begin{array}{c}0.11 \\
(0.02-0.60)\end{array}$ & $\begin{array}{c}0.17 \\
(0.05-0.51)\end{array}$ & $\begin{array}{c}0.19 \\
(0.04-0.78)\end{array}$ & $\begin{array}{c}0.35 \\
(0.09-1.20)\end{array}$ & HAIC & \\
\hline $\begin{array}{c}0.10 \\
(0.01-0.64)\end{array}$ & $\begin{array}{c}0.15 \\
(0.03-0.58)\end{array}$ & $\begin{array}{c}0.17 \\
(0.03-0.86)\end{array}$ & $\begin{array}{c}0.32 \\
(0.07-1.34)\end{array}$ & $\begin{array}{c}0.91 \\
(0.44-1.89)\end{array}$ & RT HAIC \\
\hline
\end{tabular}

Figure 6 Comparison of adverse events for regimens in advanced HCC OR $(95 \% \mathrm{Cl})$.

Note: The numbers in bold indicate $\mathrm{OR}$ and $95 \% \mathrm{Cl}$ are significant.

Abbreviations: $\mathrm{Cl}$, confidence interval; HAIC, hepatic arterial infusion chemotherapy; OR, odds ratio; SBRT, stereotactic body radiotherapy; TACE, transarterial chemoembolization; $\mathrm{RT}$, radiotherapy. 


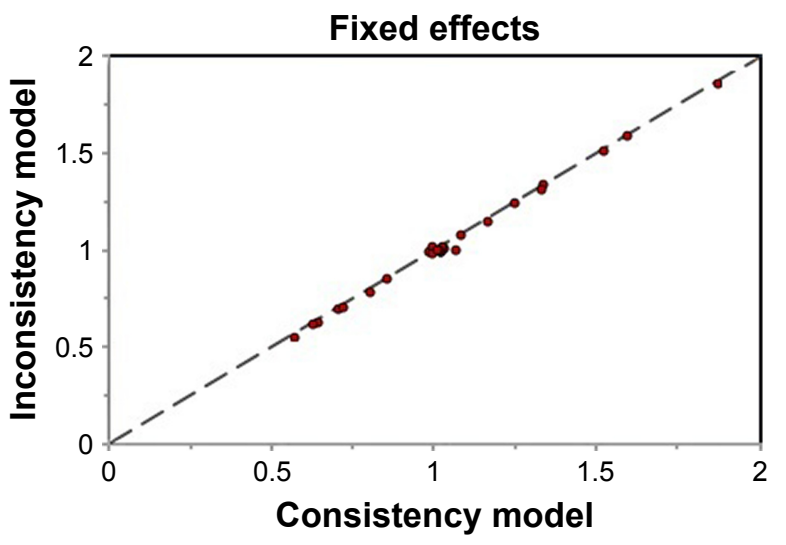

Figure 7 Network inconsistency assessment in fix-effect model.

comparisons in a network of studies using novel statistical methods. It can help us to make a quantitative comparison of the efficacy and safety of various competing treatments in a single analysis.

\section{Study limitation}

The limitations of our network meta-analysis should be considered in the interpretation of the results. First, the baseline characteristics of the enrolled studies may influence the heterogeneity and the results, such as the dose of HAIC used which depends on patients' body surface area, the location of portal vein thrombus, type of tumor, and the primary tumor size, and there were slightly differences between the studies and may be confounding factor. Second, selection bias resulting from cohort data because patients or clinical investigators may select treatment modalities depending on patients' physical condition or investigator's decision. Third, the small sample size of some studies may cause overestimation of the outcomes. However, our study has advantages that 1) the selected patients had mHCC with portal vein invasion, which may reduce heterogeneity or inconsistency and 2) the primary outcome is more comprehensive than the published conventional meta-analysis.

\section{Conclusion}

The network meta-analysis provided evidence that the combination of HAIC or TACE or sorafenib with 3D-RT improved survival and better outcome. Future randomized controlled trials are needed to confirm the advantages of combined therapy of interested modalities over those modalities used alone for $\mathrm{mHCC}$ with portal vein invasion.

\section{Disclosure}

The authors report no conflicts of interest for this work.

\section{References}

1. Ferlay J, Soerjomataram I, Ervik M, et al [webpage on the Internet]. Cancer incidence and mortality worldwide: IARC Cancer Base No. 11. Lyon, France: International Agency for Research on Cancer; 2014. Available from: http://globocan.iarc.fr. Accessed February 24, 2015.

2. Arii S, Yamaoka Y, Futagawa S, et al. Results of surgical and nonsurgical treatment for small-sized hepatocellular carcinomas: a retrospective and nationwide survey in Japan. The Liver Cancer Study Group of Japan. Hepatology. 2000;32(6):1224-1229.

3. Llovet JM, Bruix J. Systematic review of randomized trials for unresectable hepatocellular carcinoma: chemoembolization improves survival. Hepatology. 2003;37(2):429-442.

4. Ikai I, Arii S, Kojiro M, et al. Reevaluation of prognostic factors for survival after liver resection in patients with hepatocellular carcinoma in a Japanese nationwide survey. Cancer. 2004;101(4):796-802.

5. Xue TC, Xie XY, Zhang L, Yin X, Zhang BH, Ren ZG. Transarterial chemoembolization for hepatocellular carcinoma with portal vein tumor thrombus: a meta-analysis. BMC Gastroenterol. 2013;13:60.

6. Cheng SH, Lin YM, Chuang VP, et al. A pilot study of three-dimensional conformal radiotherapy in unresectable hepatocellular carcinoma. J Gastroenterol Hepatol. 1999;14(10):1025-1033.

7. Woo HY, Heo J. New perspectives on the management of hepatocellular carcinoma with portal vein thrombosis. Clin Mol Hepatol. 2015; 21(2):115-121.

8. Yin J, Bo WT, Sun J, et al. New evidence and perspectives on the management of hepatocellular carcinoma with portal vein tumor thrombus. J Clin Transl Hepatol. 2017;5(2):169-176.

9. Silva JP, Berger NG, Tsai S, et al. Transarterial chemoembolization in hepatocellular carcinoma with portal vein tumor thrombosis: a systematic review and meta-analysis. HPB (Oxford). 2017;19(8):659-666.

10. Park JY, Ahn SH, Yoon YJ, et al. Repetitive short-course hepatic arterial infusion chemotherapy with high-dose 5-fluorouracil and cisplatin in patients with advanced hepatocellular carcinoma. Cancer. 2007; 110(1):129-137.

11. Ando E, Tanaka M, Yamashita F, et al. Hepatic arterial infusion chemotherapy for advanced hepatocellular carcinoma with portal vein tumor thrombosis: analysis of 48 cases. Cancer. 2002;95(3):588-595.

12. Kim HY, Kim JD, Bae SH, et al. A comparative study of high-dose hepatic arterial infusion chemotherapy and transarterial chemoembolization using doxorubicin for intractable, advanced hepatocellular carcinoma. Korean J Hepatol. 2010;16(4):355-361.

13. Eun JR, Lee HJ, Moon HJ, Kim TN, Kim JW, Chang JC. Hepatic arterial infusion chemotherapy using high-dose 5 -fluorouracil and cisplatin with or without interferon-alpha for the treatment of advanced hepatocellular carcinoma with portal vein tumor thrombosis. Scand J Gastroenterol. 2009;44(12):1477-1486.

14. Fuss M, Salter BJ, Herman TS, Thomas CR Jr. External beam radiation therapy for hepatocellular carcinoma: potential of intensity-modulated and image-guided radiation therapy. Gastroenterology. 2004; 127(5 Suppl 1):S206-S217.

15. Andolino DL, Johnson CS, Maluccio M, et al. Stereotactic body radiotherapy for primary hepatocellular carcinoma. Int J Radiat Oncol Biol Phys. 2011;81(4):e447-e453.

16. Meng MB, Cui YL, Lu Y, et al. Transcatheter arterial chemoembolization in combination with radiotherapy for unresectable hepatocellular carcinoma: a systematic review and meta-analysis. Radiother Oncol. 2009;92(2):184-194.

17. Tazawa J, Maeda M, Sakai Y, et al. Radiation therapy in combination with transcatheter arterial chemoembolization for hepatocellular carcinoma with extensive portal vein involvement. $J$ Gastroenterol Hepatol. 2001;16(6):660-665.

18. Seong J, Park HC, Han KH, et al. Clinical results of 3-dimensional conformal radiotherapy combined with transarterial chemoembolization for hepatocellular carcinoma in the cirrhotic patients. Hepatol Res. 2003;27(1):30-35. 
19. Fujino H, Kimura T, Aikata H, et al. Role of 3-D conformal radiotherapy for major portal vein tumor thrombosis combined with hepatic arterial infusion chemotherapy for advanced hepatocellular carcinoma. Hepatol Res. 2014;45(6):607-617.

20. Onishi H, Nouso K, Nakamura S, et al. Efficacy of hepatic arterial infusion chemotherapy in combination with irradiation for advanced hepatocellular carcinoma with portal vein invasion. Hepatol Int. 2015; 9(1):105-112.

21. Chuma M, Taguchi H, Yamamoto Y, et al. Efficacy of therapy for advanced hepatocellular carcinoma: intra-arterial 5-fluorouracil and subcutaneous interferon with image-guided radiation. J Gastroenterol Hepatol. 2011;26(7):1123-1132.

22. Katamura Y, Aikata H, Takaki S, et al. Intra-arterial 5-fluorouracil/ interferon combination therapy for advanced hepatocellular carcinoma with or without three-dimensional conformal radiotherapy for portal vein tumor thrombosis. J Gastroenterol. 2009;44(5):492-502.

23. Moher D, Liberati A, Tetzlaff J, Altman DG; PRISMA Group. Preferred reporting items for systematic reviews and meta-analyses: the PRISMA statement. Int J Surg. 2010;8(5):336-341.

24. Lencioni R, Llovet JM. Modified RECIST (mRECIST) assessment for hepatocellular carcinoma. Semin Liver Dis. 2010;30(1):52-60.

25. Higgins JPT, Green S, editors. Cochrane Handbook for Systematic Reviews of Interventions Version 5.1.0 (updated March 2011). The Cochrane Collaboration; 2014. Available from: www.cochranehandbook.org

26. GRADEpro. The Cochrane IMS. Available from: http://ims.cochrane. org/revman/gradepro. Accessed November 12, 2017.

27. Choi GH, Shim JH, Kim MJ, et al. Sorafenib alone versus sorafenib combined with transarterial chemoembolization for advanced-stage hepatocellular carcinoma: results of propensity score analyses. Radiology. 2013;269(2):603-611.

28. Bai W, Wang YJ, Zhao Y, et al. Sorafenib in combination with transarterial chemoembolization improves the survival of patients with unresectable hepatocellular carcinoma: a propensity score matching study. J Dig Dis. 2013;14(4):181-190.

29. Pinter M, Hucke F, Graziadei I, et al. Advanced-stage hepatocellular carcinoma: transarterial chemoembolization versus sorafenib. Radiology. 2012;263(2):590-599.

30. Li XL, Guo WX, Hong XD, et al. Efficacy of the treatment of transarterial chemoembolization combined with radiotherapy for hepatocellular carcinoma with portal vein tumor thrombus: a propensity score analysis. Hepatol Res. 2016;46(11):1088-1098.

31. Lu DH, Fei ZL, Zhou JP, Hu ZT, Hao WS. A comparison between three-dimensional conformal radiotherapy combined with interventional treatment and interventional treatment alone for hepatocellular carcinoma with portal vein tumour thrombosis. J Med Imaging Radiat Oncol. 2015;59(1):109-114.

32. Koo JE, Kim JH, Lim YS, et al. Combination of transarterial chemoembolization and three-dimensional conformal radiotherapy for hepatocellular carcinoma with inferior vena cava tumor thrombus. Int J Radiat Oncol Biol Phys. 2010;78(1):180-187.

33. Jeong SW, Jang JY, Lee JE, et al. The efficacy of hepatic arterial infusion chemotherapy as an alternative to sorafenib in advanced hepatocellular carcinoma. Asia Pac J Clin Oncol. 2012;8(2):164-171.

34. Kawaoka T, Aikata H, Hyogo H, et al. Comparison of hepatic arterial infusion chemotherapy versus sorafenib monotherapy in patients with advanced hepatocellular carcinoma. J Dig Dis. 2015;16(9): 505-512.

35. Song DS, Song MJ, Bae SH, et al. A comparative study between sorafenib and hepatic arterial infusion chemotherapy for advanced hepatocellular carcinoma with portal vein tumor thrombosis. J Gastroenterol. 2015;50(4):445-454.
36. Kang J, Nie Q, DU R, et al. Stereotactic body radiotherapy combined with transarterial chemoembolization for hepatocellular carcinoma with portal vein tumor thrombosis. Mol Clin Oncol. 2014;2(1):43-50.

37. Sapir E, Tao Y, Schipper MJ, et al. Stereotactic body radiation therapy as an alternative to transarterial chemoembolization for hepatocellular carcinoma. Int J Radiat Oncol Biol Phys. 2018;100(1):122-130.

38. MRC Biostatistics Unit, University of Cambridge [webpage on the Internet]. Available from: https://www.mrc-bsu.cam.ac.uk/software/bugs/ the-bugs-project-winbugs/

39. Mills EJ, Thorlund K, Ioannidis JP. Demystifying trial networks and network meta-analysis. BMJ. 2013;346:f2914.

40. Salanti G, Ades AE, Ioannidis JP. Graphical methods and numerical summaries for presenting results from multiple-treatment meta-analysis: an overview and tutorial. J Clin Epidemiol. 2011;64(2):163-171.

41. Higgins JP, Thompson SG. Quantifying heterogeneity in a metaanalysis. Stat Med. 2002;21(11):1539-1558.

42. Dias S, Welton NJ, Sutton AJ, Caldwell DM, Lu G, Ades AE. Evidence synthesis for decision making 4: inconsistency in networks of evidence based on randomized controlled trials. Med Decis Making. 2013; 33(5):641-656.

43. Llovet JM, Bustamante J, Castells A, et al. Natural history of untreated nonsurgical hepatocellular carcinoma: rationale for the design and evaluation of therapeutic trials. Hepatology. 1999;29(1):62-67.

44. Huo YR, Eslick GD. Transcatheter arterial chemoembolization plus radiotherapy compared with chemoembolization alone for hepatocellular carcinoma: a systematic review and meta-analysis. JAMA Oncol. 2015;1(6):756-765.

45. Zhang L, Hu P, Chen X, Bie P. Transarterial chemoembolization (TACE) plus sorafenib versus TACE for intermediate or advanced stage hepatocellular carcinoma: a meta-analysis. PLoS One. 2014;9(6):e100305.

46. Zhao Q, Zhu K, Yue J, et al. Comparison of intra-arterial chemoembolization with and without radiotherapy for advanced hepatocellular carcinoma with portal vein tumor thrombosis: a meta-analysis. Ther Clin Risk Manag. 2016;13:21-31.

47. Tse RV, Hawkins M, Lockwood G, et al. Phase I study of individualized stereotactic body radiotherapy for hepatocellular carcinoma and intrahepatic cholangiocarcinoma. J Clin Oncol. 2008;26(4):657-664.

48. Bujold A, Massey CA, Kim JJ, et al. Sequential phase I and II trials of stereotactic body radiotherapy for locally advanced hepatocellular carcinoma. J Clin Oncol. 2013;31(13):1631-1639.

49. Cammà $\mathrm{C}$, Cabibbo $\mathrm{G}$, Petta $\mathrm{S}$, et al. Cost-effectiveness of sorafenib treatment in field practice for patients with hepatocellular carcinoma. Hepatology. 2013;57(3):1046-1054.

50. Yoon SM, Lim YS, Won HJ, et al. Radiotherapy plus transarterial chemoembolization for hepatocellular carcinoma invading the portal vein: long-term patient outcomes. Int J Radiat Oncol Biol Phys. 2012; 82(5):2004-2011.

51. Kim TH, Kim DY, Park JW, et al. Three-dimensional conformal radiotherapy of unresectable hepatocellular carcinoma patients for whom transcatheter arterial chemoembolization was ineffective or unsuitable. Am J Clin Oncol. 2006;29(6):568-575.

52. Li X, Feng GS, Zheng CS, Zhuo CK, Liu X. Expression of plasma vascular endothelial growth factor in patients with hepatocellular carcinoma and effect of transcatheter arterial chemoembolization therapy on plasma vascular endothelial growth factor level. World J Gastroenterol. 2004;10(19):2878-2882.

53. Zhang X, Wang K, Wang M, et al. Transarterial chemoembolization (TACE) combined with sorafenib versus TACE for hepatocellular carcinoma with portal vein tumor thrombus: a systematic review and meta-analysis. Oncotarget. 2017;8(17):29416-29427.

54. Keating GM, Santoro A. Sorafenib: a review of its use in advanced hepatocellular carcinoma. Drugs. 2009;69(2):223-240. 


\section{Publish your work in this journal}

Therapeutics and Clinical Risk Management is an international, peerreviewed journal of clinical therapeutics and risk management, focusing on concise rapid reporting of clinical studies in all therapeutic areas, outcomes, safety, and programs for the effective, safe, and sustained use of medicines. This journal is indexed on PubMed Central, CAS,

EMBase, Scopus and the Elsevier Bibliographic databases. The manuscript management system is completely online and includes a very quick and fair peer-review system, which is all easy to use. Visit $\mathrm{http}: / / \mathrm{www}$.dovepress.com/testimonials.php to read real quotes from published authors.

Submit your manuscript here: http://www.dovepress.com/therapeutics-and-clinical-risk-management-journal 